\title{
Nom / Nominal (Grammaire)
}

\section{Salem Chaker}

\section{OpenEdition}

\author{
Journals
}

Édition électronique

URL : https://journals.openedition.org/encyclopedieberbere/2748

DOI : 10.4000/encyclopedieberbere. 2748

ISSN : 2262-7197

\section{Éditeur}

Peeters Publishers

\section{Édition imprimée}

Date de publication : 5 octobre 2012

Pagination : 5566-5572

ISBN : 978-90-429-2718-6

ISSN : $1015-7344$

\section{Référence électronique}

Salem Chaker, « Nom / Nominal (Grammaire) », Encyclopédie berbère [En ligne], 34 | 2012, document N62, mis en ligne le 15 décembre 2020, consulté le 17 février 2022. URL : http://

journals.openedition.org/encyclopedieberbere/2748; DOI : https://doi.org/10.4000/ encyclopedieberbere. 2748

Ce document a été généré automatiquement le 17 février 2022.

(c) Tous droits réservés 


\section{Nom / Nominal (Grammaire)}

\section{Salem Chaker}

1 En grammaire berbère, on définira le Nom, comme toute autre classe, à la fois par sa combinatoire étroite (marques grammaticales obligatoires ou facultatives qui lui sont associées) et ses fonctions* syntaxiques.

\section{La combinatoire}

2 De manière parallèle au verbe*, mais beaucoup moins systématiquement du fait des emprunts massifs à des langues étrangères qui déstructurent le système, le nom peut être défini par l'association d'une racine* lexicale, d'un schème nominal et de marques obligatoires :

\begin{tabular}{|l|l|l|l|l|}
\hline & & NOM & \\
\hline Racine lexicale & $\leftrightarrow$ & Schème nominal & $\leftrightarrow$ & Marques obligatoires \\
\hline & & (de nom d'action) & & Genre : masc. /fem. \\
\hline & & (de nom concret) & & Nombre : sing. /plur. \\
\hline & & (de nom d'agent) & & Etat : libre/annexion \\
\hline & & (de nom d'instrument & & \\
\hline & & (d'adjectif) & & \\
\hline
\end{tabular}

3 Ex : amyar, "vieillard/chef », peut-être décomposé en : racine MrR, "grand/âgé »+ schème nominal (adjectif) XXaX, + préfixe $a$-, marques obligatoires (masc. + sing. + état libre). 

même statut syntaxique. Le genre* et le nombre sont des modalités ou marques du nom. On notera qu'en berbère, le genre fait bien l'objet d'un choix autonome et n'est pas inhérent au lexème. Cela est dû à la polyvalence sémantique de l'opposition masc./ fem. qui recouvre des distinctions diverses: mâle / femelle (pour les êtres sexués), grand / normal, normal / petit, collectif ou générique / individu (voir notice « Genre », G27, EB XX, 1998).

Les schèmes nominaux sont des marques amalgamées à la racine, constituées de séquences vocaliques et, éventuellement, de morphèmes dérivationnels préfixés :

- amkraz, " laboureur ", s'analyse en : racine KRZ (labourer) + schème de nom d'agent $\boldsymbol{m X X a X}$ (cf. « Dérivation », D35, EB XV, 1995).

Ces processus de dérivation sont, dans la langue actuelle, peu productifs et largement figés, même si la néologie* contemporaine leur redonne une "seconde vie ». Si en principe tout nominal berbère (non emprunté) doit pouvoir s'analyser comme l'association d'une racine et d'un schème, la réalité synchronique est bien différente : une grande majorité des nominaux sont inanalysables en synchronie parce qu'ils sont devenus des formes lexicales isolées, ne s'intégrant pas dans une série dérivationnelle,

- soit parce que la racine dont ils sont issus a (presque) totalement disparu,

- soit parce que les évolutions phonétiques ont été si importantes que leur décomposition est désormais difficile,

- soit, enfin, parce que les évolutions sémantiques ont été telles qu'il n'existe plus de lien sémantique immédiat entre les formes considérées.

Concrètement, cela signifie que pour beaucoup de nominaux, l'identification d'un schème de dérivation (et donc d'une racine) relève de l'analyse diachronique (étymologie) et non de l'analyse synchronique.

Ainsi, le kabyle amnay, "cavalier », est historiquement le dérivé sur schème de nom d'agent (a) $\boldsymbol{m X X a X}$, d'une racine $N Y$, attestée dans de nombreux dialectes (Maroc, touareg...), où le verbe ny signifie « monter (à cheval) »; amnay est donc originellement " celui qui monte à cheval, le cavalier ». Il est clair qu'en kabyle cette analyse relève de la pure diachronie et n'a plus d'existence dans la réalité synchronique - ni bien sûr dans la conscience des locuteurs - puisque ce dialecte ne connaît plus le verbe ny, remplacé par l'emprunt à l'arabe rkəb. de $/ \mathrm{m} />/ n /$ ) d'une racine $G H / W$, "témoigner ", qui a totalement disparu dans les dialectes Nord et dont il n'existe plus de trace qu'en touareg.

Lionel Galand (1974, p. 99) résumait bien cette tendance lourde en écrivant : "Chaque mot tend à vivre de sa vie propre [...]. En terme saussuriens, l'évolution fait du berbère une langue moins " grammaticale » et plus « lexicologique ». »

La marque d'état (état libre / état d'annexion; voir notice "Annexion », A226, EB V 1988), quant à elle, est déterminée par la fonction syntaxique du nominal : l'état d'annexion est l'indice d'un rapport de dépendance vis-à-vis d'un autre nominal (nom ou pronom) précédent. De ce fait, certains auteurs (anglo-saxons ou d'influence anglosaxonne) n'ont pas hésité à parler des «cas» du berbère, ce qui est quelque peu artificiel, puisque cela conduit à poser une déclinaison nominale à deux cas, très polyvalents, assez éloignée des systèmes casuels classiques (latin, grec, russe et autres langues indo-européennes, arabe...) où chaque cas correspond à une fonction 
syntaxique fondamentale assez bien délimitée. Cette opposition a connu un très fort processus de figement et est largement devenue une simple contrainte morphologique (El Moujahid 1981, 1982) ; il serait pour autant excessif de lui dénier toute fonction syntaxique en synchronie. Mais il est vrai que dans de nombreux contextes, elle est en redondance avec un autre morphème, de nombreuses catégories de noms ne la connaissent pas/plus et que les séquences et occurrences où elle reste réellement porteuse d'information sont limitées en nombre (avec quelques variations selon les dialectes). Enfin, son signifiant est inextricablement amalgamé aux autres marques du nom (genre et nombre) ; cette considération morphologique fait qu'il est plus simple de traiter ensemble toutes ces marques conjointes obligatoires du nom, plutôt que de les dissocier sur la base leurs différences de statut.

13 Au plan diachronique, il est admis par tous les berbérisants que les segments initiaux de ces marques obligatoires, dans la forme qu'on leur connait actuellement, sont secondaires et résultent d'un processus de figement de morphèmes pré-posés, à l'origine facultatifs, puis devenus préfixes obligatoires :

\begin{tabular}{|c|c|c|}
\hline$a^{-}=$déictique de proximité masculin & / & ta- déictique de proximité fem. \\
\hline$i-=$ déictique de masc. plur. (cf wi) & / & ti- déictique de fém. plur. \\
\hline (a) $m$ mar $=($ ce $>$ le $)$ vieux & $>$ & $a-m \gamma a r=$ vieux (homme) \\
\hline (ta) $m$ yar-t $=($ cette $>$ la) vieille & $>$ & $t a-m \gamma$ ar $-t=$ vieille \\
\hline (i) $m$ rar-n $=($ ces $>$ les $)$ vieux & $>$ & $i-m \gamma$ yr $-n=$ vieillards \\
\hline (ti) $m$ yar-in $=($ ces $>$ les $)$ vieilles & $>$ & ti-myar-in $=$ vieilles \\
\hline
\end{tabular}

Il s'agissait certainement d'une opposition " défini » / « indéfini (présence / absence de la marque), issue comme dans bien des langues (langues sémitiques, romanes, germaniques...) du recyclage et de la refonctionnalisation de morphèmes déictiques. On peut d'ailleurs suspecter la marque de l'état d'annexion du masculin du berbère Nord (w/u-) d'être aussi d'origine déictique puisque certains dialectiques (zénètes*) ont - $u$ au lieu de - $a$ comme forme de base du déictique de proximité. Bien entendu, cette reconstruction est purement diachronique et il n'existe plus "d'article défini » en berbère actuel - contrairement à ce qu'on peut lire dans certains ouvrages de vulgarisation - puisque ces marques, devenues obligatoires, ne s'opposent plus à leur absence. C'est bien dans ce sens, purement historique, qu'il faut comprendre la terminologie, qui peut prêter à confusion, de Werner Vycichl (1957).

Ce phénomène de figement des marques initiales du nom est sans doute très ancien, protohistorique, voire préhistorique, puisque la toponymie antique de l'Afrique du Nord fournit, à travers les notations latines, des centaines de noms à initiale $T(h)_{v}$ - et souvent à finale $-t$; ce qui semble indiquer que le système des marques initiales du nom que nous connaissons en berbère actuel était déjà largement en place au moment de la domination romaine. L. Galand (1989) a certes émis des réserves et interrogations sérieuses quant à l'interprétation que l'on peut faire de ces toponymes antiques à initiales $T(h)$-, et a notamment attiré l'attention sur la possibilité qu'ils puissent être autre chose que des noms à marque de genre féminin du berbère contemporain (par 
exemple des syntagmes à support de détermination; cf. touareg: ta məllət $=$ « celle étant blanche ", qui ne sont pas des noms mais des propositions relatives). Certes, les matériaux onomastiques* antiques sont et resteront en grande partie obscurs et les analyses largement hypothétiques, mais en l'occurrence, il existe de nombreux indices convergents qui incitent à considérer que, au moins pour partie, le système des marques actuelles, nominales et verbales, était déjà en place à époque libyque, ne seraitce que la très grande fréquence de toponymes en $T v-(t)$ sur des thèmes très courts qui ne peuvent être autre chose que des nominaux et qui, souvent, renvoient immédiatement à des formes lexicales berbères; on pense à des nom comme Thala, Thizi, Thugga, Thelepte, Thagaste... (Voir aussi notice 016 "Onomastique libycoberbère »). dire au niveau du mot. En amont, antérieurement à la mise en phrase, les unités significatives minimales ne présentent pas cette différenciation marquée: Nom et Verbe sont des complexes obtenus par adjonctions de marques spécifiques à des racines lexicales communes, indifférenciées. En d'autres termes, la dichotomie Nom / Verbe, nette au plan de la syntaxe de la phrase, cache une unité plus profonde de l'ensemble des formes constituant le lexique de la langue. Si l'on part des unités minimales, l'existence d'un système verbo-nominal unique ressort avec clarté. A partir d'un stock indifférencié de racines lexicales, on obtient, par adjonction de marques grammaticales spécifiques des Noms ou des Verbes.

Nom et verbe sont reliés de façon régulière et immédiatement apparente par un réseau de relations, sémantiques et formelles, qui les rapproche et réduit singulièrement la signification des différences combinatoires et/ou fonctionnelles. 


\section{Les autres nominaux}

23 La présentation ci-dessus a été focalisée sur le substantif qui, ayant les latitudes combinatoires et fonctionnelles les plus larges, sert d'étalon pour la définition de la classe du Nom. Mais celle-ci est en fait une macro-classe syntaxique composée de différentes sous-catégories, parmi lesquelles on peut distinguer: les sous-catégories lexicales, qui comprennent

- Les substantifs (voir ci-dessus),

- Les adjectifs*

- Les numéraux*

et les sous-catégories grammaticales, qui regroupent tous les paradigmes pronominaux :

- Pronoms* personnels

- Pronoms non personnels divers : déictiques, indéfinis, interrogatifs.

L'adjectif* partage tous les traits combinatoires et fonctionnels du substantif. Il porte lui aussi les marques de genre, de nombre et d'état et peut être prédicat de phrase nominale. Il se distingue du substantif par le fait qu'il peut, en plus, être déterminant direct (séquence immédiate, sans marque spécifique) d'un substantif :

\begin{tabular}{|l|l|l|}
\hline akal abərkan & $=$ & «(la/une) terre noire $~$ \\
\hline terre noire & & \\
\hline
\end{tabular}

L'adjectif est une sous-catégorie syntaxique, identifiée fondamentalement par sa fonction spécifique de déterminant de substantif.

Les numéraux*, outre leur appartenance à une série formelle particulière, sont identifiés par leur non-compatibilité avec deux des modalités obligatoires du substantif, le nombre et l'état. Mis à part cette restriction combinatoire, ce sont des noms de plein statut: contrairement à ce qui a lieu dans d'autres langues, le nombre berbère n'est pas un déterminant ; c'est lui qui, dans la suite :

\begin{tabular}{|l|l|l|}
\hline Nombre & $\leftarrow$ & Nom \\
\hline yiwn & & wrgaz \\
\hline un & & homme (+ E.A.) \\
\hline
\end{tabular}

est le déterminé, et le nom subséquent le déterminant (la subordination étant marquée par l'état d'annexion) :

\begin{tabular}{|l|l|l|}
\hline Nombre & $\leftarrow$ & Nom \\
\hline déterminé & & déterminant (« un de homme »). \\
\hline
\end{tabular}


Les pronoms* constituent plusieurs sous-systèmes clos que l'on a intérêt à intégrer à la sphère du nom, ceci malgré la nécessaire intervention de critères non-syntaxiques (énonciation) pour leur identification. Les caractéristiques énonciatives des pronoms (notamment les personnels) n'en font pas des êtres extra-syntaxiques : leur intégration dans les énoncés se fait sur la base des mêmes types de contraintes paradigmatiques et syntagmatiques que celle de toute autre unité non pronominale :

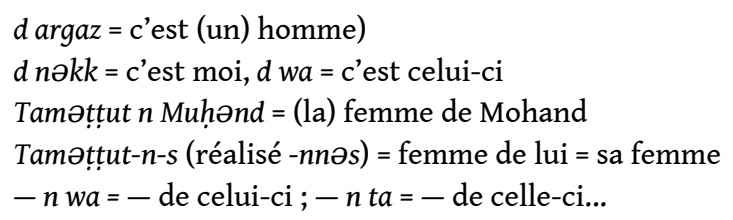

30 Sur le plan syntaxique, ce sont bien des noms comme les autres, quelles que soient par ailleurs leurs spécificités pragmatique et sémantique et tous ces substituts assument des fonctions typiquement nominales, y compris celle de prédicat de phrase nominale, comme l'illustrent les parallélismes des séquences ci-dessus.

$31 \rightarrow$ Voir aussi notices: (A56) «Adjectif », EB II, 1985 ; (A226) « Annexion », EB V, 1988; (F35) « Fonctions », EB XIX, 1997 ; (G27) « Genre », EB XX, 1998 ; « Pronoms ».

\section{BIBLIOGRAPHIE}

BASSET A., 1952 (1969) - La langue berbère, Oxford/Londres.

BENTOLILA F., 1981 - Grammaire fonctionnelle d'un parler berbère, Paris, SELAF.

BENVENISTE E., 1966 et 1974 - Problèmes de linguistique générale, Paris, NRF-Gallimard, I \& II.

CANTINEAU J., 1950 - « Racine et schème », Mélanges William Marçais, Paris, Maisonneuve, p. 119-124.

CHAKER S., 1983 - Un parler berbère d'Algérie (Kabylie) : syntaxe, Université de Provence.

CHAKER S., 1984 - Textes en linguistique berbère (Introduction au domaine berbère), Paris, CNRS.

CHAKER S., 1989 - « Lexicographie et comparaison : « le dictionnaire informatisé de la langue berbère » ", Journée de linguistique berbère, Paris, Inalco.

COHEN D., 1968 - « Les langues chamito-sémitiques », Le langage, Paris, NRF-Gallimard (« La Pléiade »).

DIAKONOFF I. M., 1988 - Afrasian languages, Moscou, Nauka.

ELMOUJAHID E., 1981 - La classe du nom dans un parler de la langue tamazight : le tachelhiyt d'Igherm (Sous-Maroc), thèse de doctorat de $3^{\mathrm{e}}$ cycle, Université de Paris-V.

ELMOUJAHID E., 1982 - « Un aspect morphologique du nom en tamazight : l'état d'annexion », Langues et Litteratures (Rabat), 2.

GALAND L., 1974 - « Signe arbitraire » et signe motivé en berbère », Congrès International de Linguistique Sémitique et Chamito-Sémitique (Paris, 1969), La Haye/Paris, Mouton. 
GALAND L., 1984 - « Le comportement des schèmes et des racines dans l'évolution de la langue : exemples touaregs », Current Progress in Afro-asiatic Linguistics (= Proceedings of the third International Hamito-Semitic Congress), Amsterdam, John Benjamins publishing Company.

GALAND L., 1988 - « Le berbère ", Les langues dans le monde ancien et moderne, $3^{\mathrm{e}}$ partie : Les langues chamito-sémitiques, Paris, CNRS.

GALAND L., 1989 - « « T(h) » in Libyan and Canarian place-names », Almogaren, XX/1, p. 32-41.

GALAND-PERNET P., 1959 - « Nom et verbe en berbère », Travaux de l'Institut de Linguistique de Paris, 4, p. 35-37.

GARDE P., 1981 - « Des parties du discours, notamment en russe », BSLP, LVXVI, fasc. 1, p. 155-189.

GARDE P., 1983 - «Présupposés linguistiques de la théorie des parties du discours », Travaux du Cercle linguistique d'Aix-en-Provence, 1, p. 1-8.

PRASSE K.-G., 1972-74 - Manuel de grammaire touarègue (tahaggart), Copenhague, Akademisk Forlag, 1974 : IV-V, Nom.

TAÏFI M., 1988 - « Problèmes méthodologiques relatifs à la confection d'un dictionnaire $\mathrm{du}$ tamazight ", Awal, 4.

TAÏFI M., 1990 - « Pour une théorie des schèmes en berbère », Etudes et Documents Berbères, 7.

VYCICHL W., 1957 - «L'article défini en berbère », Mémorial André Basset, Paris, Adrien

Maisonneuve, p. 139-146. Version résumée dans Etudes et documents berbères, 1, p. 61-63.

\section{INDEX}

Mots-clés : Ethnologie, Linguistique 